\title{
Evaluation of cutting temperature of a tool with a curved cutting edge
}

\author{
Denis Moiseev ${ }^{1, *}$, Anatoliy Ryzhkin ${ }^{1}$ and Gennadiy Volokitin ${ }^{2}$ \\ ${ }^{1}$ Don State Technical University, «Metal-cutting machines and tools» department, 344000 Rostov-on-Don, Russian Federation \\ ${ }^{2}$ Don State Technical University, «Mathematics» department, 344000 Rostov-on-Don, Russian Federation
}

\begin{abstract}
A model for solving the problem of heat distribution along the front surface of a tool with a shaped profile is considered. Analytical dependencies for estimating the temperature of the tool of elliptic profile for turning with radial feed in Cartesian and polar coordinate systems are obtained for the model under consideration. As a result, the obtained dependences can be applied to similar thermal calculations of instruments with other forms of plane curves both in turning with radial feed and in milling conditions. The calculation of a flat temperature field on the front surface of a shaped tool has been carried out and it has revealed areas with the highest temperatures at the contact area and the maximum wear values.
\end{abstract}

\section{Introduction}

Thermal state of the tool, working on forced modes, affects the rate of its wear. The levels of the temperatures while cutting can reach critical values, therefore the problem of evaluation of heat flows and temperatures using calculation methods is actual. Dependencies for calculation of the cutting temperatures for instruments with a straight cutting edge are known [1-3], but those dependencies can be used for the problem in a linear formulation, when it is impossible to determine the temperature distribution at any point on the front surface of the tool.

A shaped cutter of an elliptic profile is presented in the article as an object of the research, which can be used both for modeling of process of radial shaped turning and for milling.

\section{Problem formulation and solution methods}

The problem of distribution of the temperature field is solved on the front surface of the convex part of the cutter of the elliptical profile (region 2-3-4 in Fig. 1, a), because the cutting conditions will be practically identical for a concave part (regions 1-2 and 4-5) of the tool in view of the fact that the convex and the concave parts are the generating curves of the same elliptic profile.

The origin of coordinates for a convex part of the elliptic cutter with semiaxis $a$ and $b$ is located in the centre of the tool (system $X_{I} O_{I} Y_{I}$ ). For this case the equation of a convex part of ellipse in polar coordinates $r$ and $\theta$ (Fig. 1, b) in the system $X_{I} O_{l} Y_{l}$ has the following form:

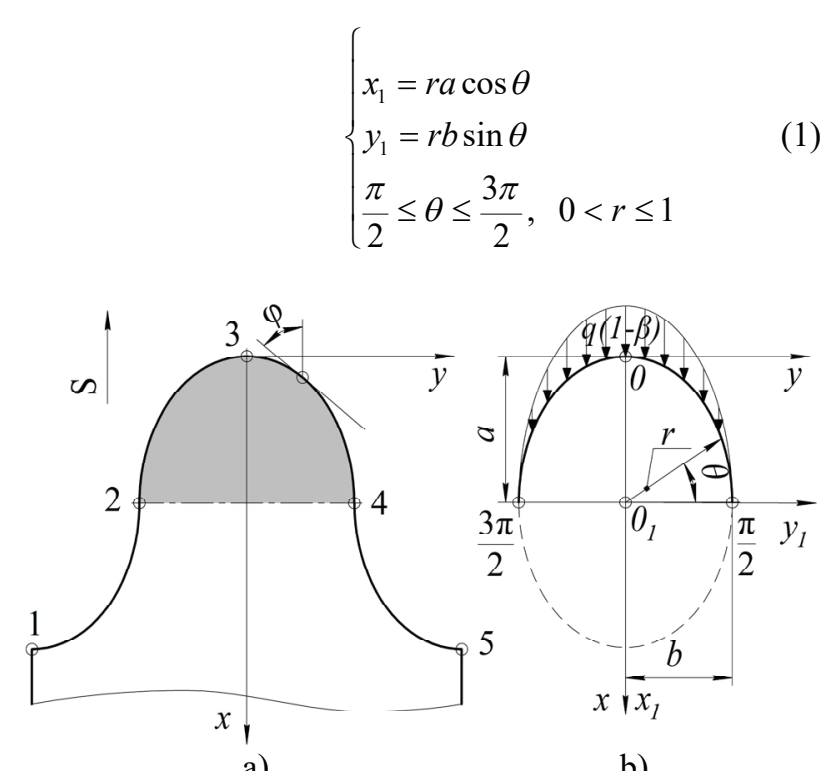

a)

Fig. 1. The profile of the tool (a) and the scheme of heat flows (b)

For the $X O Y$ system, the ellipse equation will have the form:

$$
\left\{\begin{array}{l}
x=a(1+r \cos \theta) \\
y=r b \sin \theta
\end{array}\right.
$$

The transformation formulas from polar coordinates to Cartesian $x, y$ have the form:

\footnotetext{
* Corresponding author: denisey2003@mail.ru
} 


$$
\left\{\begin{array}{l}
r^{2}=\frac{(x-a)^{2}}{a^{2}}+\frac{y^{2}}{b^{2}}, 0 \leq r \leq 1 \\
\theta=\operatorname{arctg}\left(\frac{a-x}{y}\right)+\frac{\pi}{2}, n p u \frac{\pi}{2} \leq \theta \leq \pi \\
\theta=\operatorname{arcctg}\left(\frac{x-a}{y}\right)+\pi, n p u \pi \leq \theta \leq \frac{3 \pi}{2}
\end{array}\right.
$$

We assume that the heat flow with $q_{0}$ density acts on the tool during cutting (Fig. 1, b). It propagates along the cutting blade unevenly and assumes the maximum value on the top of the tool (point 3 in Fig. 1, a), and approaching the points 2 and 4 it decreases and becomes equal to zero at these points. Let then the heat flow along the cutting blade vary according to the dependence:

$$
f(\theta)=\frac{1}{\pi^{2}}\left(-4 \theta^{2}+8 \pi \theta-3 \pi^{2}\right),
$$

from which the heat flow density function along the cutting blade takes the form:

$$
q(\theta)=(1-\beta) \cdot q_{O} \cdot f(\theta)
$$

$f(\theta)$ is a normalized heat flow distribution function at the boundary; $(1-\beta)$ is a coefficient that takes into account the proportion of heat flowing into the tool.

This type of heat flow can be explained by the inconstancy of cutting geometry: rear angle of the tool takes maximum value on the top 3 (Fig.1,a), which provides the best cutting conditions; when approaching points 2 and 4 it decreases and becomes equal to 0 , i.e. the cutting process becomes impossible.

The stationary heat equation for the conditions, described above, has the following form:

$$
\Delta T-m_{1} T=0
$$

where $\Delta$ is the Laplace operator; $m_{1}>0$ - coefficient that takes into account the heat exchange of the instrument with the environment.

In the polar coordinate system with the origin in the center of the tool, the differential heat equation has the form:

$$
\begin{aligned}
& \frac{1}{r} \frac{\partial}{\partial r}\left(r \frac{\partial T}{\partial r}\right)+\frac{1}{r^{2}} \frac{\partial^{2} T}{\partial \theta^{2}}-m_{1} T=0, \\
& T=T(x, y)=T(r, \theta)
\end{aligned}
$$

with the following boundary conditions:

$$
\begin{aligned}
& \left.\frac{\partial T}{\partial r}\right|_{\substack{r=1 \\
\frac{\pi}{2} \leq \theta \leq \frac{3 \pi}{2}}}=\frac{q(\theta)}{\lambda_{1}} \\
& T\left(r, \frac{\pi}{2}\right)=T\left(r, \frac{3 \pi}{2}\right)=0, T(0, \theta)<\infty
\end{aligned},
$$

$\lambda_{1}$-coefficient of thermal conductivity of the tool material.

The solution of the boundary value problem (7), (8) has been found using the method of separation of variables:

$$
T=Y(r) \Phi(\theta)
$$

Substituting (9) into (7), we obtain [4]:

$$
\frac{r^{2} Y^{\prime \prime}+r Y^{\prime}-m_{1} r^{2} Y}{Y}=-\frac{\Phi^{\prime \prime}}{\Phi}=v^{2}
$$

where $v=$ const .

The dependence (10) has 2 solutions:

$$
\begin{gathered}
\Phi^{\prime \prime}+v^{2} \Phi=0, \Phi \neq 0 \\
r^{2} Y^{\prime \prime}+r Y^{\prime}-\left(m_{1} r^{2}+v^{2}\right) Y=0, \quad Y \neq 0
\end{gathered}
$$

The variables are also separated in the boundary conditions. It follows from (8):

$$
\Phi\left(\frac{\pi}{2}\right)=\Phi\left(\frac{3 \pi}{2}\right)=0
$$

The solution of the boundary-value problem (11), (13) has the following form:

$$
\begin{aligned}
& \Phi_{v}(\theta)=\Phi_{n}(\theta)=A_{n} \cos n \theta+B_{n} \sin n \theta \\
& (v=n, n \in Z)
\end{aligned}
$$

Using the substitution $\rho=\sqrt{m_{1}} r$ and the equality $v=n$, we obtain from (12) an ordinary differential equation:

$$
\rho^{2} Y^{\prime \prime}+\rho Y^{\prime}-\left(\rho^{2}+n^{2}\right) Y=0, n \in Z
$$

Dependence (15) is a Bessel equation of diffusion type, which defines the modified functions: the Bessel function of the imaginary argument and the modified Hankel function (MacDonald function) [5]. If the argument $n$ is also valid, then both functions take real values.

Since the solution of the problem is limited when $\rho=0$, due to the singularities at zero of the functions of MacDonald we get:

$$
Y_{n}(\rho)=C_{n} I_{n}(\rho)
$$

Consequently: 


$$
\begin{aligned}
& T_{n}(r, \theta)=Y_{n}(\rho) \Phi_{n}(\theta)= \\
& =I_{n}\left(\sqrt{m_{1}} r\right)\left(A_{n} \cos n \theta+B_{n} \sin n \theta\right)
\end{aligned}
$$

Using the principle of superposition, the solution of the boundary value problem is:

$$
T=\sum_{n=0}^{\infty} I_{n}\left(\sqrt{m_{1}} r\right)\left(A_{n} \cos n \theta+B_{n} \sin n \theta\right)
$$

The coefficients $A_{n}, B_{n}$ in the expression (18) can be determined from the boundary condition (8).

Using the expansion of expression (4) in the Fourier series and the boundary conditions (8), we obtain formulas for the coefficients $A_{n}\left(B_{n}=0\right)$ and as a result, we obtain a solution of the boundary value problem for the thermal field of the tool in polar coordinates [6].

$$
\begin{aligned}
& T=\frac{(1-\beta) q_{0}}{3 \lambda_{1} \sqrt{m_{1}} I_{0}^{\prime}\left(\sqrt{m_{1}}\right)} I_{0}\left(\sqrt{m_{1}} r\right)+ \\
& +\sum_{k=1}^{\infty}\left[I_{k}\left(\sqrt{m_{1}} r\right)\left(\frac{(1-\beta) q_{0}}{\lambda_{1} \pi^{2} \sqrt{m_{1}}}\right)\left(\frac{a_{k}}{I_{k}^{\prime}\left(\sqrt{m_{1}}\right)}\right) \cos k \theta\right]
\end{aligned}
$$

Using the dependencies (3) and (19) we obtain the final expression for the tool temperature field in Cartesian coordinates:

$$
\begin{aligned}
& T=\frac{(1-\beta) q_{0}}{\lambda_{1} \sqrt{m_{1}}}\left\{\frac{I_{0}\left[\sqrt{m_{1} \cdot\left(\frac{(x-a)^{2}}{a^{2}}+\frac{y^{2}}{b^{2}}\right)}\right]}{3 I_{0}^{\prime}\left[\sqrt{m_{1}}\right]}+\right. \\
& +\sum_{k=1}^{\infty} \frac{\left[m_{1}\left(\frac{(x-a)^{2}}{a^{2}}+\frac{y^{2}}{b^{2}}\right)\right]^{\frac{1}{2}}\left(\frac{-\pi \cos \frac{\pi k}{2}}{k^{2}}-\frac{2 \sin \frac{\pi k}{2}}{k^{3}}\right)}{I_{k}^{\prime}\left[\sqrt{m_{1}}\right]} . \\
& \left.\cdot \frac{8 I_{k}}{\pi^{3}} \cdot \cos \left[k\left(\operatorname{arctg} \frac{a-x}{y}+\frac{\pi}{2}\right)\right]\right\}
\end{aligned}
$$

$I_{k}$ is a Bessel function of an imaginary argument of the $k$-th order.

To calculate the temperature of the tool according to the equations (19) and (20) we must have the number of original data:

- heat flow $q_{0}=0,252 \cdot \sigma \cdot \frac{V}{K_{a}}[7-8]$.

- $\quad$ the distribution coefficient of the heat flow $\beta$.

According to the solution presented in [1], the distribution coefficient of heat flow is equal to:

$$
\beta=\frac{\lambda_{2}}{\lambda_{2}+\lambda_{1} \sqrt{m_{1}} \cdot \sqrt{1,5 a_{2} \tau}},
$$

where: $\lambda_{2}-$ the thermal conductivity coefficient of the material of the workpiece; $\sigma-$ the tensile strength of the workpiece material; $a_{2}-$ the thermal diffusivity coefficient of the workpiece material; $\tau$-the contact time of the chip with the front surface of the tool equal to [1]:

$$
\tau=\frac{l_{k}}{V_{c}}=\frac{2 S \cdot \sin \varphi \cdot K_{a}\left[K_{a}(1-\operatorname{tg} \gamma)+\frac{1}{\cos \gamma}\right]}{V}
$$

in dependence (22): $l_{k}$ - length of the chip contact with the front surface of the tool; $S$ - feed value; $K_{a}-$ chip shrinkage ratio; $\varphi$ - the main angle in plan of the tool; $\gamma$ - front angle of the tool; $V$-cutting speed.

The heat flow distribution coefficient is a function of the variables $K_{a}, \gamma, \varphi$ which take into consideration the inconstancy of the tool geometry (this approach is considered in [2]). Therefore, it is necessary to know the values of these parameters at each point of the cutting blade.

The functional dependences of the parameters entering into (21) - (22) are the following:

- for this case we assume the value of the front angle $\gamma$ to be constant and equal to $0^{\circ}$ :

the dependence of the chip shrinkage coefficient $K_{a}$ for the processing of steel 45 with a carbide cutter of grade T15K6 has been obtained experimentally in the following form:

$$
K_{a}=0,462 \cdot V^{0,414} \cdot e^{-0,433 V} \cdot S^{-0,238} \cdot \varphi^{-0,09},
$$

where cutting speed $V$ has the dimension $\mathrm{m} / \mathrm{s}$, feed $S$ - $\mathrm{m} / \mathrm{rev}$, and the angle in plan $\varphi-\mathrm{rad}$.

- the value of the angle in the plan $\varphi$ can be determined from the equation of the ellipse in the $X O Y$ coordinate system (Fig. 1,a), using the derivative of the function $y=\frac{b}{a} \sqrt{2 a x-x^{2}} \quad[6]$.

$$
\varphi=\operatorname{arctg}\left(y^{\prime}\right)=\operatorname{arctg} \frac{b(a-x)}{a^{2} \sqrt{1-\frac{(x-a)^{2}}{a^{2}}}}
$$

Thus, on the basis of dependences (21) - (23) it can be concluded that the heat flow distribution coefficient in this case is a function dependent on the main angle in plan; therefore, in calculating the heat leaving the tool, the position of the point on the cutting tool blade should be considered. 


\section{Example of calculation of the thermal distribution of the tool}

The calculation of the thermal field of the cutter of the elliptical profile while turning with a radial feed of steel 45 with a carbide $\mathrm{T} 15 \mathrm{~K} 6$ cutter is presented below:

Initial data:

Cutting modes: $S=3 \cdot 10^{-4} \mathrm{~m} / \mathrm{rev} ., V=3 \mathrm{~m} / \mathrm{s}$

Parameters of the ellipse: $a=4,5 \cdot 10^{-3} \mathrm{~m}, b=5 \cdot 10^{-3} \mathrm{~m}$

Tool (hard alloy T15K6): $\lambda_{1}=27,2 \mathrm{~W} / \mathrm{m} \cdot$.gr., $m_{1}=40 \mathrm{~m}^{-1}$

Workpiece (steel 45): $\sigma=598 \mathrm{MPa}$, $a_{2}=8 \cdot 10^{-6} \mathrm{~m}^{2} / \mathrm{s}$., $\lambda_{2}=40,1 \mathrm{~W} / \mathrm{m} \cdot \mathrm{gr}$.

Given the coordinate values within the front surface of the tool $(x \in[0 ; a] \quad$ and $y \in\left[-b \sqrt{1-\frac{(x-a)^{2}}{a^{2}}} ; b \sqrt{1-\frac{(x-a)^{2}}{a^{2}}}\right]$, we obtain the temperature distribution (Fig.2).

Fig. 2 shows the distribution of temperatures along the front surface of the tool in the form of level lines.

Fig. 3 shows the distribution of temperatures along the front surface of the tool in sections $A-A, B-B, \ldots E-E$, cutting the tool by planes parallel to the $O X$ axis with an increment of $\Delta=1 \cdot 10^{-3} \mathrm{~m}$.

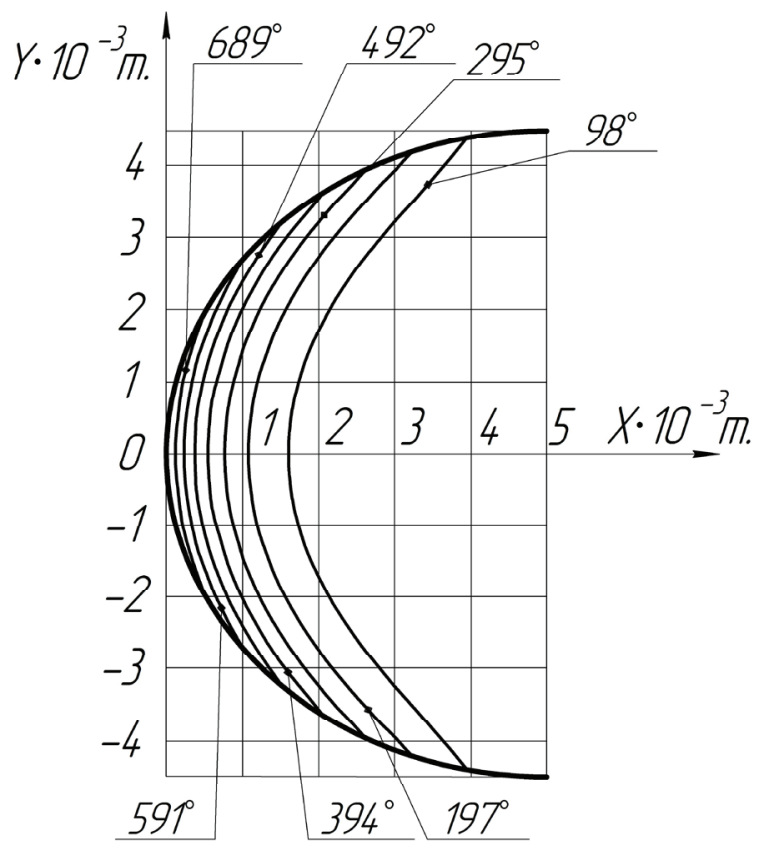

Fig.2. Temperature field on the front surface of the tool

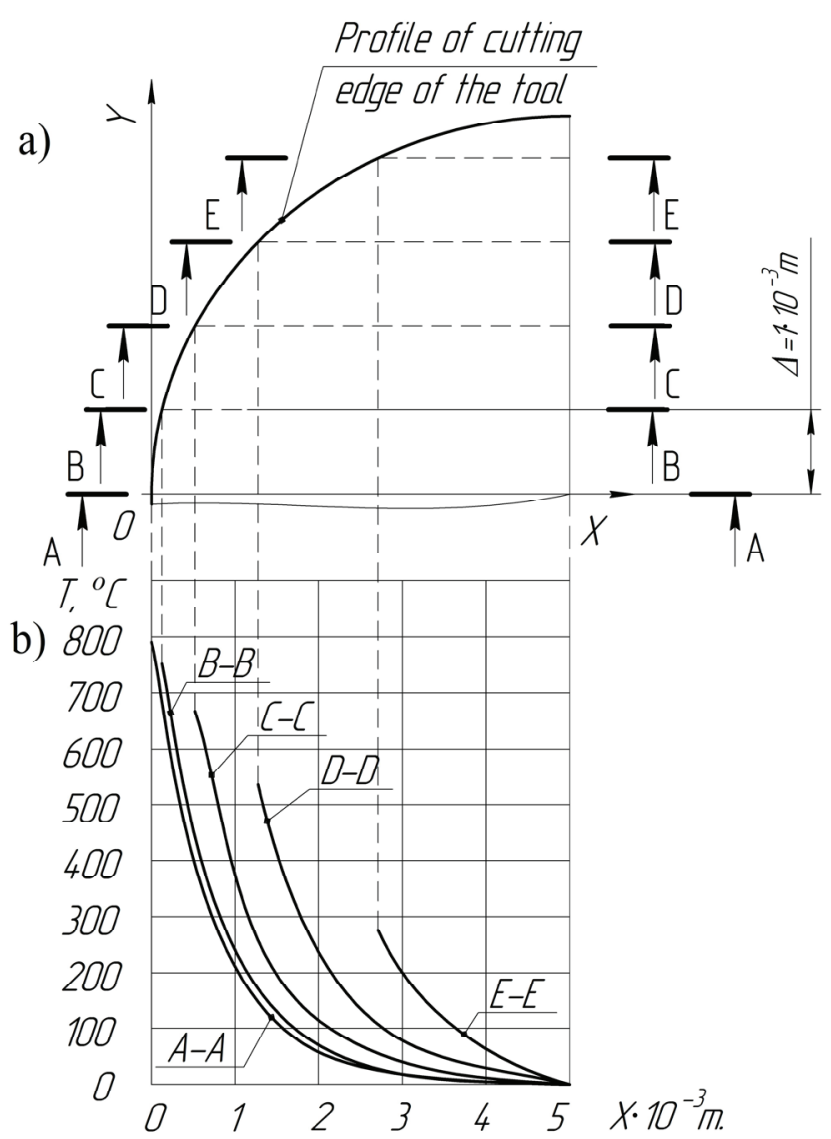

Fig.3. The tool profile (a) and the corresponding temperature distribution (b) in sections parallel to the tool axis

As we can see from the obtained diagrams, the temperature assumes minimum values far from the cutting edge and increases as it approaches it. The maximum temperature value, equal to 788 degrees, is observed on the top of the tool, where the angle in plan and the rear angle take the maximum values (point with coordinates $x=0, y=0$ ).

\section{Conclusions}

The solution of the problem of heat propagation on the working surface of the tool allows to evaluate the distribution pattern and the level of emerging temperatures on the front surface of the instrument, to identify areas of possible maximum wear and to calculate the wear rate at any point of the blade, and to predict the tool's durability.

\section{References}

1. A.A. Ryzhkin, K.G. Shuchev, M.M. Klimov, Obrabotka materialov rezaniem (Fenix, Rostov-onDon, 2008) [in Russian].

2. A.A. Ryzhkin, D.V. Moiseev, Vestnik DSTU. Temperature evaluation under curved-edge tool cutting, 29-36 (2014) [in Russian]. 
3. A.A. Ryzhkin, Teplofizicheskie protsessy pri iznashivanii instrumental'nykh rezhushhikh materialov (Izd. tsentr DGTU, Rostov-on-Don, 2005) [in Russian].

4. A.N. Tikhonov, A.A. Samarskiy, Uravneniya matematicheskoy fiziki (Nauka, Moscow, 1977) [in Russian].

5. E. Yanke, F. Emde, F. Lesh, Spetsialnyie funktsii (Nauka, Moscow,1964) [in Russian].
6. I.N. Bronshtein, K.A. Semendyaev, Spravochnik dlya inzhenerov $i$ uchaschihsya vtuzov (Nauka, Moscow, 1986) [in Russian]

7. A.N. Reznikov, Teplofizika rezaniya (Mashinostroyenie, Moscow,1969) [in Russian].

8. A.N. Reznikov, Teplofizika protsessov mekhanicheskoj obrabotki materialov (Mashinostroyenie, Moscow, 1981) [in Russian]. 